between onset and weakness. Supportive findings include: antecedent infection, CSF albumino-cytological dissociation, neurophysiological evidence of neuropathy, and presence of IgG anti-GT1a or anti-GQ1b antibodies. Brain MRI may be indicated to exclude brainstem ischemia, inflammation or brain tumor.

\title{
References.
}

1. Nagashima T, et al. Arch Neurol. 2007 Oct;64(10):1519-23.

2. Ropper AH. Arch Neurol. 1986 Nov;43(11):1150-2.

\section{RECURRENT MILLER FISHER SYNDROME}

Investigators from University of Siena, Italy, describe 2 children with recurrent Miller Fisher syndrome. Episodes occurred at age 11.5 and 13 years in Patient 1 and at age 8 and 13 years in Patient 2. Both patients responded to treatment with steroids. Patient 1 presented with diplopia, unsteady gait and clumsiness. Neurologic examination showed ataxia, hyporeflexia, and ophthalmoplegia. Treatment with iv immunoglobulin was effective initially, but failed to prevent progressive weakness during the second attack that subsequently responded to steroid therapy. Patient 2 presented with paresthesia of hands and diplopia, ataxia, paresis of $6^{\text {th }}$ and $7^{\text {th }}$ cranial nerves, muscle weakness, and hyporeflexia. Recovery from both the initial and second attack followed steroid therapy. (Grosso S, Verrotti A, Tei M, Cornacchione S, Giannini F, Balestri P. Recurrent Miller Fisher syndrome in children. Pediatr Neurol 2014 Mar;50(3):269-71).

COMMENTARY. Recurrent Miller Fisher syndrome [1] is rare in childhood, and the second attack may be more aggressive and resistant to therapy. Steroids may be indicated if iv immunoglobulin is ineffective [2].

\section{References.}

1. Fisher M. N Engl J Med. 1956 Jul 12;255(2):57-65.

2. Toru S, et al. Muscle Nerve. 2012 May;45(5):763-4.

\section{PARANEOPLASTIC DISORDERS}

\section{PARANEOPLASTIC DISORDERS AND OVARIAN TUMORS}

Researchers in Chang Gung University, Kaohsiung, and National Cheng Kung University, Tainan, Taiwan, assessed the prevalence and spectrum of paraneoplastic neurological disorders (PND) in children with benign ovarian tumor and the long-term outcome. The charts of 133 female patients below 18 years of age diagnosed with a pathologically proven benign ovarian tumor, Jan 1993 - Dec 2010, were reviewed, mostly mature teratoma. Six patients $(4.5 \%)$ had neuropsychiatric manifestations, the majority (5) with onset after age 10 years. Depression or low mood, headache, mutism, hypoventilation, seizures, hallucination, vomiting and hypersalivation were the most common symptoms. NMDAR encephalitis in 2 patients and acute disseminated encephalomyelitis in 1 partially resolved after tumor removal and immunotherapy. One patient not receiving immunotherapy had neurological sequelae and long ICU stay. (Hsu 
M-H, Huang C-C, Hung P-L, et al. Paraneoplastic neurological disorders in children with benign ovarian tumors. Brain Dev 2014 Mar;36(3):248-53).

COMMENTARY. Paraneoplastic disorders (PND) present with multiple manifestations resembling subacute encephalitis, peripheral neuropathy, cerebellar ataxia, opsoclonus myoclonus with neuroblastoma, and other symptoms. Patients with antiNMDAR encephalitis may present with psychosis, memory deficits, seizures, speech problems, involuntary movements, and breathing disorders. Approximately 50\% cases have ovarian tumors, mostly teratoma. In the present study, 5 of $6(83 \%)$ patients with PND and ovarian teratoma had complete remission of symptoms after tumor removal. The authors recommend immunotherapy in all patients following tumor removal, despite apparent recovery after surgery [1].

References.

1. Hsu M-H, et al. Brain Dev. 2014 Mar;36(3):248-53.

\section{DEMYELINATING DISORDERS}

\section{POTASSIUM CHANNEL KIR4.1-SPECIFIC ANTIBODIES AND ACQUIRED DEMYELINATING DISEASE}

Researchers at Technische Universitat, Munich, and other centers in Germany, studied the prevalence of KIR4.1-IgG by ELISA in 47 children with acquired demyelinating disease (ADD), in 22 with other neurologic diseases, 22 with autoimmune disease, and in 18 healthy controls. Serum antibodies to KIR4.1 were identified in $57 \%$ of children with ADD but none with other neurologic disease, autoimmune disease or healthy controls. KIR4.1-IgG titers were predominantly found in children with MS or clinically isolated syndrome and in 1 in 3 with demyelinating encephalitis, similar to the prevalence in adults with ADD. KIR4.1-IgG titers were significantly higher in children with ADD compared with control groups $(\mathrm{p}<0.0001)$; they were not age-dependent and did not correlate with myelin oligodendrocyte glycoprotein (MOG) antibody responses. MOG-IgG occurs before age $10 \mathrm{y}$ in ADD whereas KIR4.1 antibodies are found in older children and adult patients with MS. (Kraus V, Srivastava R, Kelluri SR, et al. Potassium channel KIR4.1-specific antibodies in children with acquired demyelinating CNS disease. Neurology 2014 Feb 11;82(6):470-3).

COMMENTARY. KIR4.1, a potassium channel expressed on oligodendrocytes and astrocytes, contributes to the maintenance of the electrochemical gradient by removing potassium from the extracellular space. Mutations of the KIR4.1 gene cause EAST syndrome characterized by epilepsy, ataxia, sensorineural deafness, and tubulopathy [1]. The prevalence of KIR4.1-IgG in children with MS is similar to adults.

\section{References.}

1. Bockenhauer D, et al. N Engl J Med. 2009 May 7;360(19):1960-70. 